\title{
Support at Decision in Electrical Systems of subtransmission through selection of Topologies by a Paraconsistent Simulator
}

\author{
J. I. Da Silva Filho, Member, IEEE, J. M. Camargo, M. R. Santos, A. S. Onuki, M. C. Mario, L. F. P. \\ Ferrara, D. V. Garcia, J. M. C. Pereira, Member, IEEE and A. Rocco, Member, IEEE
}

\begin{abstract}
In this paper, we present a Simulator Program that identifies the topologies and supports operation staff in decisionelectrical power system operation consider load reestablishment procedures and topological possibilities to each event, offering a selection of the best settings. Facing a electrical power transmission system contingency, the Simulator considers the current state and uses special algorithms that detect the grid topology, interprets results and presents to the operators a rank of procedures with their respective degrees of reliability. For these actions the software Simulator make the circuits breakers states analysis and electric keys, load flow solution with the on line data, makes risk prediction and the mathematical analysis of remote sensing values. The software Simulator uses in some of his actions, special algorithms that are based on Paraconsistent Annotated Logic (PAL), which is a non-classical logic whose main property is to accept contradiction in their fundamentals. These algorithms based on PAL offer greater speed of processing and allows the Paraconsistent Simulator of topologies (ParaSimTop) to be implemented in real time.
\end{abstract}

Keywords - simulator, algorithms, paraconsistent logic, expert system, electrical power system.

\section{INTRODUÇÃO}

A IMPLANTAÇÃO de novas tecnologias em Sistemas de distribuição e subtransmissão de Energia Elétrica possibilitaram a aquisição e o armazenamento de grandes quantidades de dados de informação em tempo real [1]. Os sistemas de supervisão e aquisição de dados (SCADASupervisory Control and Data Acquisition) instalados nas subestações das redes de distribuição das concessionárias são

J. I. Da Silva Filho, Universidade Santa Cecília (UNISANTA), Santos, São Paulo, Brasil,inacio@unisanta.br

J. M. Camargo, AES-Eletropaulo SA, São Paulo, SP, Brasil, jose.camargo@AES.com

M. R. Santos, AES-Eletropaulo SA, São Paulo, SP, Brasil, marcos.rosa@aes.com

A. S. Onuki, Universidade Santa Cecília (UNISANTA), Santos, São Paulo,Brasil, shozost@yahoo.com.br

M. C. Mario, Universidade Santa Cecília (UNISANTA), Santos, São Paulo,Brasil,cmario@unisanta.br

L. F. P. Ferrara, Universidade Santa Cecília (UNISANTA), Santos, São Paulo,Brasil,1fpferrara@uol.com.br;

D. V. Garcia, Universidade Santa Cecília (UNISANTA), Santos, São Paulo, Brasil, dora@unisanta.br

J. M. C. Pereira, INESC/Porto - Instituto de Engenharia de Sistemas e Computadores do Porto, cidade do Porto, Portugal, jpereira@inescporto.pt

A. Rocco, Universidade Santa Cecília (UNISANTA), Santos, São Paulo, Brasil, a.rocco@terra.com.br partes desta tecnologia e assim apresentam grande capacidade de aquisição e armazenam enormes quantidades de sinais de informação sobre o estado e topologia da rede elétrica a cada instante [2,3]. Os dados aquisitados continuamente pelo SCADA são armazenados e ficam disponíveis para as análises e, devido a enorme quantidade de informação fica impossível de serem interpretados sem o apoio computacional [2]. A fase de análise e interpretação dos dados é muito importante na formação de tomadas de decisão e deve ser efetuada com algoritmos capazes de oferecer nível de confiabilidade suficiente para que ações posteriores apresentem os efeitos esperados.

Apesar da moderna tecnologia empregada, por diversos motivos, os quais em muitas das vezes fogem do controle devido a existência de variáveis complexas dentro dos processos de fabricação dos dispositivos sensores, são gerados erros de vários tipos na grande massa de dados aquisitados pelo SCADA [3,4]. Frente a isso muitos dados se apresentam com informações incompletas, ambíguas, vagas e contraditórias. Portanto, na realidade, mesmo em tecnologias recentes as incertezas geradas por estes sinais que representam informações incertas e inconsistentes estão bastantes presentes nos dados armazenados [5].

Considerando estas condições de incertezas e a necessidade de ferramentas computacionais capazes de apoiar de modo eficiente as ações da equipe da Operação de sistema de subtransmissão de energia elétrica, apresentamos neste trabalho um software Simulador de apoio as decisões que seleciona topologias adequadas para cada estado atual da rede. Para alcançar esta finalidade o software Simulador primeiramente promove a aquisição e faz analises em dados provenientes de grandezas elétricas do sistema, que são armazenados pelo SCADA. Considerando a necessidade de oferecer tratamento especial aos dados incertos provenientes do SCADA empregamos, em partes principais que compõem o Software Simulador, alguns algoritmos especiais que são estruturados em uma lógica não clássica, denominada de Lógica Paraconsistente Anotada com anotação de dois valores (LPA2v) [3][6-8].

De maneira geral as Lógicas Paraconsistentes têm como principal propriedade a consideração da contradição em seus fundamentos, sem trivialização, isto é, sem que o peso dos conflitos gerados pelos dados contraditórios possam invalidar as conclusões das analises [3]. Portanto, a Lógica Paraconsistente faz parte do grupo de lógicas que se opõem as leis estritamente binárias da lógica clássica, com ênfase na 
derrogação do principio da não contradição. Devido a essa característica especial, com a sua aplicação em engenharia, principalmente na forma da LPA2v, são conseguidos algoritmos capazes de resolver situações complexas e com tempo de computação que não compromete a eficiência do processo de analise [7]. Apesar de serem recentes as aplicações da LPA2v em grandes Banco de Dados, e em trabalhos ligados ao SCADA utilizado em Sistemas Elétricos de Potencia, os resultados encontrados nestas aplicações são bastantes promissores e a capacita na estruturação e construção de Simuladores paraconsistentes bastante eficientes. Detalhes sobre os fundamentos teóricos e a formalização da LP podem ser encontrados em [6-8].

Neste trabalho, o Software Simulador Paraconsistente de seleção de topologias (ParaSimTop) foi construído com algoritmos especiais que fazem a aquisição de valores de grandezas elétricas nos Banco de Dados do Sistema SCADA e, através de processos matemáticos convencionais e não convencionais, como os fundamentados em LPA2v [8], aplicam tratamento computacional extraindo informações, representadas por Graus de Evidência, extremamente importantes para subsidiar tomadas de decisão em sistemas elétricos de potencia [8-10].

\section{SOFTWARE SIMULADOR PARACONSISTENTE DE TOPOLOGIAS- PARASIMTOP - VISÃO GERAL}

$\mathrm{Na}$ sua parte funcional o Simulador Paraconsistente (ParaSimTop) é um modelo computacional com a capacidade de fazer aquisição e efetuar um tratamento de dados com agregação de qualidade aos processos porque utiliza técnicas não convencionais. Analisando valores relacionados as grandezas elétricas de um sistema de distribuição de energia o Simulador é capaz de dar como resposta qual a melhor manobra, diante de uma situação de contingência ou iminente ação de remanejamento do operador.

De maneira geral, na estrutura do ParaSimTop os valores aquisitados do SCADA recebem tratamento de validação que identificam a topologia atual da rede, em seguida são acionados processos de análises com fluxos de carga, obtidos os perfis de previsões de carga e os resultados, transformados em graus de evidência, passam por algoritmos da LPA2v que fazem analises de riscos de sobrecarga. Estes procedimentos, que serão detalhados a seguir, geram dados com resultados suficientes que identificam para a equipe de Operação do Sistema de Subtransmissão os tipos de topologias mais convenientes para as possíveis mudanças bem como uma sequencias de remanejamento mais confiáveis.

Conforme visto anteriormente, em um Sistema de Subtransmissão de Energia Elétrica supervisionado pelo SCADA existem os erros de medições que são inerentes a própria complexidades de manuseio e de limites tecnológicos dos sensores $[4,5][10]$. Além da grande quantidade de dados disponíveis, em várias situações existe também a necessidade de que decisões sejam tomadas pela equipe de Operação da rede elétrica em tempo real, pois caso não haja atuação o problema pode se agravar com o sistema sendo levado a um estado critico, ou mesmo a queda total $[4,5][11]$. Para maior eficiências nestes processos se exigem implantações de programas computacionais capazes de dar tratamento conveniente e com bastante rapidez. Na Fig. 1 é mostrado um diagrama do ParaSimTop com os seus principais blocos.

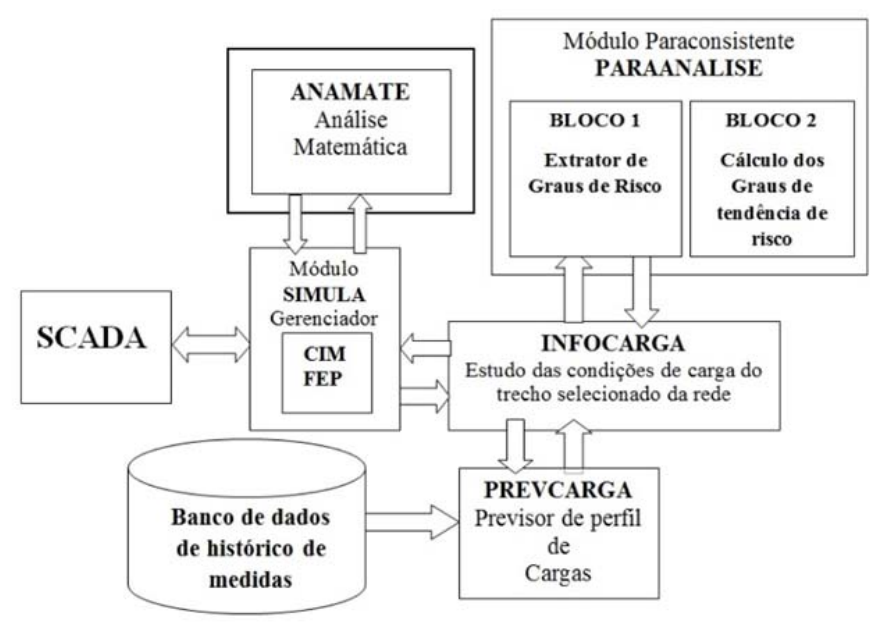

Figura 1. Diagrama em blocos do Software Simulador de seleção de Topologias (ParaSimTop).

No seu funcionamento básico o Simulador Paraconsistente utiliza resultados de estudos de fluxo, previsões de cargas e simulações de manobras para gerar informações de apoio a decisão. O apoio a Operação é oferecido elencando manobras para otimizar o remanejamento como também listando informações sobre consequências de eventuais manobras ou remanejamentos na rede.

Considerando o diagrama de blocos visto na Fig. 1, de maneira geral ao ser acionado o ParaSimTop ativa uma ação no Módulo SIMULA que é o Gerenciador de todo o processo. O gerenciamento é feito apresentando os seguintes passos:

a) Inicialmente o sistema SIMULA irá fazer a leitura da topologia elétrica da rede do trecho escolhido como padrão de testes através do módulo CIM (Common Information Model) que é um modelo de protocolos para o desenvolvimento e a integração entre os sub-sistemas seguindo todas as normas padronizadas para sistemas elétricos- e; b) do módulo FEP (Frontal End Processor) - que tem como principal função fornecer os estados do equipamento e sua respectiva medida para que seja combinado com o modelo CIM -, sendo este responsável pela definição dos estados e medidas de cada equipamento dessa topologia.

$\mathrm{O}$ processo tem início quando o operador faz uma requisição ao sistema SIMULA, informando a necessidade de avaliação de um evento (contingência ou simulação); por exemplo, quais manobras são viáveis tecnicamente para serem efetuadas para aquele evento. 1 - O módulo de estudo das condições de carga (INFOCARGA) faz uma avaliação dessa requisição, informando qual o trecho da rede afetado pela manobra, além do limite de potência permitido. Essa análise é baseada pelos valores disponibilizados pelo módulo Previsor de Carga (PREVCARGA). 2 - O bloco responsável pela análise paraconsistente (PARAANALISE) indica o Grau de Risco e a tendência do Grau de risco para este instante. 3 - As informações do item 2 mais as informações do CIM- 
Topologia e FEP-Medidas, são disponibilizadas ao módulo de analise Matemática (ANAMATE), que por sua vez informa, qual é a melhor manobra a ser efetuada pelo operador. $4-\mathrm{O}$ resultado do módulo de análise Matemática (ANAMATE) é disponibilizado ao operador pelo próprio sistema SCADA, através do módulo de comunicação FEP.

$\mathrm{Na}$ estrutura do Simulador paraconsistente, os cálculos de Previsão de Perfil de Cargas, e o método de análise de riscos de sobrecarga, são procedimentos computacionais baseados em algoritmos fundamentados em Lógica Paraconsistente Anotada com anotação de dois valores (LPA2v) [8-11]. Portanto, a seguir serão dados maiores detalhes sobre a LPA2v, bem como as formas de interpretação matemática utilizadas para a criação dos algoritmos.

\section{TRATAMENTO DE DADOS COM A LÓGICA PARACONSISTENTE}

Quando construídos com algoritmos baseados em lógica clássica, que é totalmente fundamentada em rígidas leis binárias, as incertezas que se apresentam nos dados aquisitados em um Sistema Elétrico de Potência criam uma tarefa extremamente difícil para gerar eficiência aos programas computacionais [10]. Considerando a incapacidade da lógica clássica de tratar diretamente situações retratadas por dados originados de informações contraditórias, procurou-se neste trabalho utilizar novas formas de tratamento de dados baseadas em um tipo de lógica não-clássica denominada de Lógica Paraconsistente (LP) [6]. Sendo a propriedade principal da $L P$ aceitar contradição em seus fundamentos, uma interpretação em um Reticulado de quatro vértices, na qual se considera dois valores evidenciais relacionados a uma determinada proposição $P$ permite a extração de equações. Dessa forma, a partir de um reticulado, conhecido como Reticulado de Hasse, é possível associar um tipo de Lógica Paraconsistente denominada de Lógica Paraconsistente Anotada com anotação de dois valores (LPA2v) [7,8]. A Fig. 2 mostra o Reticulado associado à LPA2v.

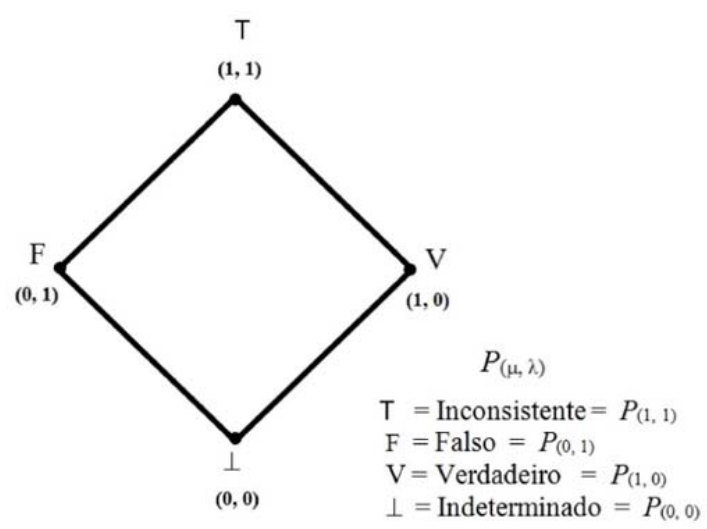

Figura 2. Reticulado de quatro Vértices associado à LPA2v.

\section{A. Principais equações da Lógica Paraconsistente Anotada com anotação de dois valores LPA2v}

A aplicação da LPA2v está ligada a uma análise matemática no Reticulado associado da LPA, visto na Fig. 2, o que resulta em uma transformação linear, conforme a equação (1). Maiores detalhes podem ser vistos em [6] e [7,8]:

$$
T(X, Y)=(x-y, x+y-1)
$$

Relacionando os componentes da transformação $T(X, Y)$ conforme a nomenclatura usual da LPA2v, onde:

$x=\mu \rightarrow$ Grau de Evidência favorável, com $0 \leq \mu \leq 1 \mathrm{e}$

$y=\lambda \rightarrow$ Grau de Evidência desfavorável, com $0 \leq \lambda \leq 1$, vem que: a) do primeiro termo obtido no par ordenado da equação da transformação fica: $x-y=\mu-\lambda \rightarrow$ o qual será denominado de grau de Certeza $\left(\mathrm{G}_{\mathrm{C}}\right)$. Portanto, o Grau de certeza é obtido por:

$$
G_{C}=\mu-\lambda
$$

b) do segundo termo obtido no par ordenado da equação da transformação fica: $x+y-1=\mu+\lambda-1 \rightarrow \quad 0$ qual será denominado de grau de Contradição $\left(\mathrm{G}_{\mathrm{ct}}\right)$. Portanto, o grau de Contradição é obtido por:

$$
G_{c t}=\mu+\lambda-1
$$

Com o $\mathrm{G}_{\mathrm{ct}}$ pode-se encontrar o seu valor normalizado, denominado de grau de Contradição normalizado $\left(\mu_{\mathrm{ctr}}\right)$ [10]. Portanto:

$$
\mu_{c t r}=\frac{G_{c t}+1}{2}
$$

A partir de um estudo mais detalhado visto em [10] pode-se encontrar o grau de Certeza Real $\left(\mathrm{G}_{\mathrm{CR}}\right)$ como um valor projetado no eixo dos graus de certeza do Reticulado através das equações: $G_{C R}=1-\sqrt{\left(1-\left|G_{C}\right|\right)^{2}+G_{c t}{ }^{2}} \quad$ se $G_{C}>0$

$$
G_{C R}=\sqrt{\left(1-\left|G_{C}\right|\right)^{2}+G_{c t}^{2}}-1 \text { se } G_{C}<0
$$

Com o $\mathrm{G}_{\mathrm{CR}}$ pode-se encontrar o seu valor normalizado, denominado de grau de Evidência Resultante $\left(\mu_{\mathrm{ER}}\right)$ [10]. Portanto:

$$
\mu_{E R}=\frac{G_{C R}+1}{2}
$$

Considerando a complexidade do problema e seguindo essa linha de raciocínio utilizou-se nesse projeto uma nova forma de concepção na construção de sistemas computacionais onde em partes fundamentais do projeto construtivo do simulador o tratamento e as análises de sinais de informações usam os conceitos da LPA2v.

\section{B. Algoritmos da Lógica Paraconsistente Anotada com anotação de dois valores LPA2v}

Com base em diversos trabalhos sabemos que algoritmos construídos a partir dos fundamentos da LPA2v se apresentam com menor tempo de resposta, facilitando ações que exigem tomadas de decisão em tempo real, como é o caso de intervenções feitas por equipes operacionais nos sistemas de subtransmissão de energia elétrica. Dessa forma, alguns Blocos do ParaSimTop, como o Previsor e o gerador de graus de Evidência de Risco, utilizam algoritmos fundamentados em Lógica Paraconsistente Anotada com anotação de dois valores-LPA2v.

A partir das equações (2) (3) (4) e (5) foram obtidos três algoritmos principais que agem no tratamento de dados do ParaSimTop. O primeiro algoritmo é denominado NAP- Nó de Analise Paraconsistente e tem como finalidade efetuar uma analise em dois sinais normalizados denominados de grau de 
Evidência favorável $(\mu)$ e grau de Evidência desfavorável $(\lambda)$, ambos relacionados à proposição $P$ que estiver em analise. $\mathrm{O}$ algoritmo NAP é descrito a seguir.

\section{B.1 .Algoritmo NAP- Nó de Analise Paraconsistente}

1. Entre com os valores de Entrada

$\mu^{*} /$ Grau de Evidência favorável $0 \leq \mu \leq 1$

$\lambda * /$ Grau de Evidência desfavorável $0 \leq \lambda \leq 1$

2. Calcule o grau de Certeza

$$
G_{C}=\mu-\lambda
$$

3. Calcule o grau de Contradição

$$
G_{c t}=(\mu+\lambda)-1
$$

4. Calcule a distância d

$$
d=\sqrt{\left(1-\left|G_{C}\right|\right)^{2}+G_{c t}^{2}}
$$

5. Calcule o grau de Contradição normalizado

$$
\mu_{c t r}=\frac{\mu+\lambda}{2}
$$

6. Determine o sinal da Saída

Se $d>1$, então faça: $S 1=0,5$ : Indefinição e vá

para o item 10

Senão vá para o próximo item

7. Determine o grau de Certeza real

$$
\begin{array}{ll}
\text { Se } G_{C}>0 & G_{C R}=(1-d) \\
\text { Se } G_{C}<0 & G_{C R}=(d-1)
\end{array}
$$

8. Calcule o grau de Evidência resultante real

$$
\mu_{E R}=\frac{G_{C R}+1}{2}
$$

9. Apresente os resultados na saída

$$
\text { Faça } S 1=\mu_{E R}
$$

\section{Fim}

O resultado do NAP é um valor denominado de grau de Evidência Resultante que sendo um valor normalizado serve como representante de evidência para análises posteriores e assim podem-se criar extensas redes de NAPs capazes de efetuar análises em grandes bancos de dados.

A rede de NAPs utilizada no ParaSimTop consiste de um algoritmo denominado de Extrator de Efeitos da Contradição (ParaExtract). O ParaExtract [7][10] recebe um grupo de sinais e, independentemente de outras informações externas tem a função fazer uma análise paraconsistente em seus valores subtraindo os efeitos causados pela contradição. O ParaExtract irá apresentar na saída um único grau de Evidência resultante real representativo do grupo. A seguir é apresentado o algoritmo ParaExtract utilizado no processo de extração de efeitos da contradição em dados do SCADA.

\section{B.2.Algoritmo Extrator de Efeitos da Contradição}

1.Apresente os valores de graus de Evidência do grupo em estudo: $\mathrm{G} \mu_{\mathrm{est}}=\left(\mu_{\mathrm{A}}, \mu_{\mathrm{B}}, \mu_{\mathrm{C}, \ldots,}, \mu_{\mathrm{n}}\right) * /$ Graus de Evidência

$$
0,0 \leq \mu \leq 1,0 * /
$$

2. Selecione o maior valor entre os graus de Evidência do grupo em estudo: $\mu_{\operatorname{maxA}}=\operatorname{Max}\left(\mu_{\mathrm{A}}, \mu_{\mathrm{B}}, \mu_{\mathrm{C}, \ldots,}, \mu_{\mathrm{n}}\right)$

3. Selecione o menor valor entre os graus de Evidência do grupo em estudo: $\mu_{\min A}=\operatorname{Min}\left(\mu_{\mathrm{A}}, \mu_{\mathrm{B}}, \mu_{\mathrm{C}, \ldots}, \mu_{\mathrm{n}}\right)$

4. Faça a análise Paraconsistente entre os valores selecionados $\mu_{\operatorname{maxA}}$ e $\mu_{\min A}: \mu_{\mathrm{R} 1}=\mu_{\operatorname{maxA}} \diamond \mu_{\operatorname{minA}} * /$ Utilização do NAP */
5. Acrescente $\mathrm{o}$ valor obtido $\mu_{\mathrm{R} 1}$ no grupo em estudo excluindo deste os dois valores $\mu_{\max }$ e $\mu_{\min }$ selecionados anteriormente: $\mathrm{G} \mu_{\mathrm{est}}=\left(\mu_{\mathrm{A}}, \mu_{\mathrm{B}}, \mu_{\mathrm{C}, \ldots}, \mu_{\mathrm{n}}, \mu_{\mathrm{R} 1)}-\left(\mu_{\max \mathrm{A}}, \mu_{\min \mathrm{A}}\right)\right.$ 6. Retorne ao item 2 até que o Grupo em estudo tenha um único elemento: $\mathrm{G} \mu_{\mathrm{est}}=\left(\mu_{\mathrm{ER}}\right) * /$ valor resultante das análises*/.

No ParaSimTop o algoritmo ParaExtract tem como função fazer o tratamento de dados recebidos do Sistema SCADA e através de sua aplicação extrair os efeitos das contradições existentes, de modo que, dos vários valores aplicados em suas entradas, apenas um valor representante do grupo, e já subtraído do efeito da contradição, seja apresentado na saída. Dessa forma o sistema é capaz de apresentar sinais depurados das incertezas e das contradições existentes nos banco de dados, sem perder informações que seriam desprezadas por algoritmos clássicos.

O terceiro algoritmo utilizado no ParaSimTop é o Analisador de tendência de risco (ParaRiskAnalisador). No ParaSimTop o algoritmo ParaRiskAnalisador tem a função de analisar dados referentes as grandezas elétricas do sistema de subtransmissão de Energia e enviar para o módulo que utiliza analises matemáticas e técnicas de IA dois valores resultantes; um em forma de grau de risco, e o outro em forma de grau de tendência de risco. Estes dois valores são gerados a partir da analise feita pelo ParaRiskAnalisador em três medições consecutivas; $\mu_{n}$-atual; $\mu_{n-1}$-anterior e $\mu_{n+1}$-posterior. $\mathrm{O}$ algoritmo ParaRiskAnalisador é descrito a seguir.

\section{B.3.Algoritmo Analisador de risco}

1. Os valores normalizados $\mu_{n}$ e $\mu_{n+1}$ são inseridos em um NAP (Nó de Análise Paraconsistente), tendo como resultado a variável $\mu_{E R}$ (grau de Evidência resultante do NAP).

Considera-se $\mu_{E R}$ como o grau de Risco.

2. Calcula-se o grau de Contradição $\left(G_{c t}\right)$.

$$
G_{c t}=\left(\mu_{n}+\mu_{n+1}\right)-1
$$

3. Calcula-se o grau de Contradição Normalizado $\left(\mu_{c t r}\right)$.

$$
\mu_{c t r}=\frac{\left(G_{c t}+1\right)}{2}
$$

4. Se $\mu_{E R}>\mu_{n}$, é calculado $x=\left(\mu_{n+1}-\mu_{c t r}\right)+\mu_{n-1}$ e feita a modelagem com variação linear e diretamente proporcional e universo de discurso com os valores $\mu_{n-1}=a_{1}$ e $\mu_{n}=a_{2}$.

4.a) Calcule o grau de tendência de risco por:

$$
\mu_{\text {tend }}= \begin{cases}0 & \text { se } x<a_{1} \\ 1 & \text { se } x>a_{2} \\ \frac{x-a_{1}}{a_{2}-a_{1}} & \text { se } x \in\left[a_{1}, a_{2}\right]\end{cases}
$$

4.b) Sinalize esse resultado com sinal positivo $\left(\mu_{\text {tend (+) }}\right)$ informando o grau de tendência de risco crescente e vá para o item 7.

5. Se $\mu_{E R}<\mu_{n}$, é calculado $x=\mu_{n-1}-\left(\mu_{n}-\mu_{c t r}\right)$ e feita a modelagem com variação linear e indiretamente proporcional e universo de discurso com os valores $\mu_{n}=a_{1}$ e $\mu_{n-1}=a_{2}$.

5.a) calcule o grau de tendência de risco por: 


$$
\mu_{\text {tend }}= \begin{cases}0 & \text { se } x>a_{2} \\ 1 & \text { se } x<a_{1} \\ \frac{x-a_{2}}{a_{1}-a_{2}} & \text { se } x \in\left[a_{1}, a_{2}\right]\end{cases}
$$

5.b) Sinalize esse resultado com sinal negativo $\left(\mu_{\text {tend }(-)}\right)$, informe o grau de tendência de risco decrescente e vá para o item 7.

6. Se $\mu_{E R}=\mu_{n}$, o grau de tendência de risco é igual a zero.

\section{Fim}

No processo de analise os graus de Evidencia de risco são informações que servirão de evidencias para a elaboração do elenco das possíveis topologias e manobras oferecidas a Operação na condição em que foi solicitada a simulação.

\section{SIMULAÇÕES E RESULTADOS}

Todos os algoritmos extraídos da LPA2v utilizados no ParaSimTop foram construídos e validados com base em um criterioso estudo anterior sobre o sistema de subtransmissão de energia elétrica onde estes seriam aplicados. Primeiramente um trecho da rede foi escolhido como padrão de teste onde se estudou mais diretamente sobre as consequências oriundas de diversas manobras suportando diferentes condições operativas, tanto em relação a topologias quanto em relação a medidas das grandezas elétricas obtidas em tempo real através do SCADA. $\mathrm{O}$ trecho definido como padrão da rede Piloto onde foram testados e validados os módulos do ParaSimTop trata-se de uma ilha referente a duas linhas de transmissão alimentadas por dois Terminais SE conforme, ilustrada na Fig. 3. O sistema é alimentado em $88 \mathrm{kV}$ e possui seis estações de distribuição, sendo duas configuradas como "padrão 76" e quatro como " padrão barra dupla" além de uma estação de consumidor.

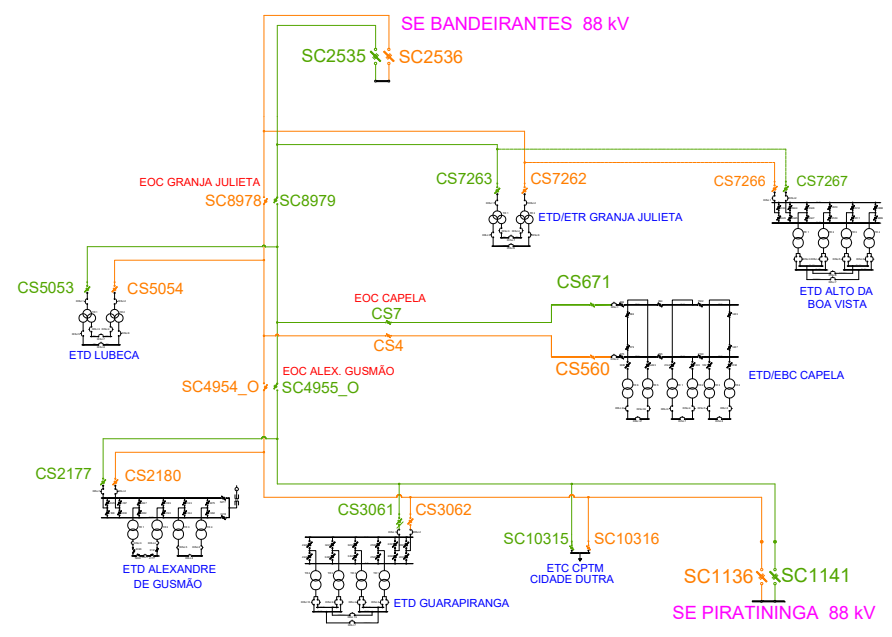

Figura 3. Trecho da rede de distribuição de Energia elétrica escolhido como piloto para os testes e validações dos algoritmos do ParaSimTop.

Inicialmente foram feitos os levantamentos de fluxos de potência e inúmeras previsões de carga baseadas em dados históricos da grande quantidade de informações de medidas. Com base nestes procedimentos para os testes foram definidas quatro contingências no sistema em estudo, onde, para cada contingência foram consideradas seis topologias de recomposição do sistema em três regimes de operação. As variáveis originadas por estas condições no trecho em teste apresentado na Fig. 3 são descritas a seguir:

A contingência 01 é referente a falta de alimentação na linha 03 proveniente da SE B. Para simular tal contingência foi considerado no modelo a abertura da chave seccionadora SC2535.

A contingência 02 é referente a falta de alimentação na linha 04 proveniente da SE P. Para simular tal contingência foi considerado no modelo a abertura da Chave seccionadora SC1136.

A contingência 03 é referente a inoperância de um trecho de linha entre as seccionadoras SC8978, CS5054, CS4 e SC4954. Para simular tal contingência foi considerado no modelo a abertura das Chaves seccionadoras que delimitam o trecho em questão.

A contingência 04 é referente a inoperância de um trecho de linha entre as seccionadoras SC2535, CS7263, CS7267 e SC8979. Para simular tal contingência foi considerado no modelo a abertura das Chaves seccionadoras que delimitam o trecho em questão.

Nos testes iniciais foram estabelecidos os casos com as condições de que sempre existirão seis opções de recomposição para cada caso de contingência. Portanto, para cada contingência descrita anteriormente, foram utilizadas seis topologias para recomposição do sistema, todas elaboradas sob os critérios de manter a operacionalidade do sistema de distribuição (tendo como premissa a possibilidade de fornecimento de energia a todas as cargas), onde foram simuladas manobras com as Chaves Seccionadoras das Terminais (Ses), Estações de Chaves (EOCs), Estações de Distribuição (ETDs) e as Estações de Consumidor (ETCs).

Também ficaram definidos três regimes de carga nas estações de distribuição e consideradas cinco datas para o estudo das contingências. Foram realizados os estudos levando em consideração três níveis de operação e em cinco dias (26, 27, 28, 29 e 30 de Abril de 2013) e como resultado destes estudos iniciais, para os procedimentos de testes e otimização do ParaSimTop o trecho escolhido correspondente gerou a 34 configurações topológicas com manobras, classificadas segundo critérios técnicos em um trabalho conjunto com a equipe de Operação do sistema de subtransmissão de energia da rede piloto.

O funcionamento básico das simulações pode ser descrito através da sequencia a seguir: 1- Inicialmente uma ação de simulação para determinado trecho ou ilha do sistema subtransmissão de energia elétrica é acionado pelo Operador que seleciona a Simulação para uma das 34 possíveis configurações topológicas típicas. Após esta ação o processo de seleção das configurações Topológicas típicas do sistema em estudo é dado inicio apoiado nas duas ferramentas de computação CIM e FEP. Nesta etapa o CIM consegue:

a) identificar no Sistema Elétrico Total da Rede de 34 Configurações Topológicas típicas diferentes.

b) para cada uma das 34 configurações Topológicas típicas diferentes o CIM consegue identificar os estados das chaves e 
Disjuntores e, portanto do funcionamento da Configuração Topológica selecionada em tempo real.

O FEP por sua vez, consegue deixar disponível em tempo real os valores das amplitudes e ângulos das grandezas elétricas relacionadas Configuração Topológica selecionada.

Após a seleção e a disponibilidade dos resultados o ParaSimTop busca os resultados de estudos das 34 Configurações Topológicas possíveis para o trecho do Sistema de Potencia acionado. E assim, com a disponibilidade destes resultados, o ParaSimTop constrói as possíveis formas de conexão frente as prováveis restrições existentes, estabelecendo-se um padrão de manobras otimizadas. $\mathrm{Na}$ construção de um Padrão de Manobras o ParaSimTop seleciona-as em dois tipos: a)Manobras Típicas de Operação; b)Manobras Típicas de Restabelecimento (recomposição).

O processo de Fluxo de cargas e Previsão para diferentes manobras selecionadas providencia dados de informação para o Sistema Especialista, que assim elabora as analises matemáticas.

O resultado da análise é representado através das manobras e tipos de manobras. A Fig. 4 mostra um dos resultados finais em tela do computador com a atuação do ParaSimTop sugerindo manobras através das informações das chaves para mudanças das possíveis topologias selecionadas.

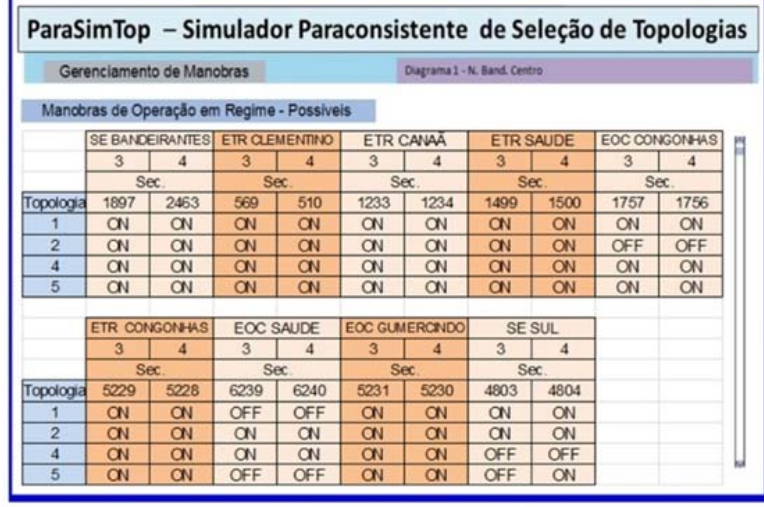

Figura 4. Resultado da simulação do ParaSimTop mostrando as possíveis manobras para mudar a topologia da rede.

Para cada topologia selecionada, uma analise de fluxo de carga é efetuada seguida de uma Previsão de carga, considerando informações provenientes de um Banco de dados histórico.

Em paralelo a previsão e estudo de fluxo, são utilizados algoritmos desenvolvidos com base nos conceitos da Lógica Paraconsistente (LPA2v) para ser feita uma análise dos valores de corrente e de tensão no trecho da rede selecionado. Esta ação gera os graus de evidência de risco cujos valores são enviados ao módulo que realiza os cálculos matemáticos. Este Módulo utiliza técnicas de IA com análises multivariáveis, e define em tempo real, através de um score, um elenco das 6 possíveis Topologias para a condição encontrada no trecho do Sistema de Potencia.

A Fig. 5 mostra um dos resultados finais em tela no qual o ParaSimTop oferece um elenco das 6 possíveis topologias para a ação da equipe de Operação.
ParaSimTop - Simulador Paraconsistente de Seleção de Topologias Diagrama 1 - N. Band centro

Topologia de Recomposição

\begin{tabular}{|c|c|c|c|c|c|c|c|c|c|c|}
\hline \multirow{2}{*}{ Topologia } & \multicolumn{2}{|c|}{ SE PI } & EOCA. GUSMÃo & \multicolumn{2}{c|}{ SECAL } & \multicolumn{2}{c|}{ EOC GJU } & \multicolumn{2}{c|}{ SE BAN } \\
\cline { 2 - 10 } & $\begin{array}{c}\text { Disj. L3 } \\
1141\end{array}$ & $\begin{array}{c}\text { Disj. L4 } \\
1136\end{array}$ & $\begin{array}{c}\text { Disj. L3 } \\
4955\end{array}$ & $\begin{array}{c}\text { Disj. L4 } \\
4954\end{array}$ & Disj. L3 & Disj. L4 & Disj. L3 & Disj. L4 & Disj. L3 & Disj. L4 \\
\hline 5 & ON & ON & ON & ON & ON & ON & OFF & OFF & OFF & ON \\
\hline 6 & ON & ON & ON & OFF & ON & ON & OFF & ON & OFF & ON \\
\hline 7 & ON & ON & ON & ON & OFF & ON & OFF & OFF & OFF & ON \\
\hline 8 & ON & ON & ON & OFF & OFF & ON & OFF & ON & OFF & ON \\
\hline 9 & ON & ON & ON & ON & ON & OFF & OFF & OFF & OFF & ON \\
\hline 10 & ON & ON & ON & OFF & ON & OFF & OFF & ON & OFF & ON \\
\hline
\end{tabular}

Figura 5. Elenco de Topologias resultantes da analise efetuada pelo ParaSimTop.

\section{A. Análise de eficiência do ParaSimTop}

Após as validações de cada bloco algorítmico do ParaSimTop foram efetuadas verificações para medir a sua eficiência na classificação de topologias frente a contingências capazes de se apresentar no trecho do sistema de distribuição de energia elétrica considerado como piloto. Estes testes de verificação foram feitos juntos a operação e obteve-se $70 \%$ de acertos quando comparados as opiniões dos técnicos da operação do sistema. Verificou-se que a porcentagem de compatibilidades não foi maior devido técnica utilizada para o calculo do fluxo de carga que se utilizou valores aproximados na determinação das tensões e correntes para ganhar em rapidez no tempo de processamento, pois um tempo longo inviabilizaria a operação de classificar topologias em tempo hábil para tomada de decisão. Verificou-se que o calculo do Fluxo de Carga apresentou variações em erros de até $2 \%$ em casos extremos, em contra partida, o tempo de processamento do cálculo em questão foi pequeno, na faixa de 50 a 70 milissegundos utilizando-se um computador de configuração básica. Com estes arredondamentos nos valores a previsão é que, caso sejam necessário a realização de 1000 simulações, o tempo gasto para apresentar os resultados será de aproximadamente de 50 a 70 segundos, o que mesmo representando um período razoavelmente curto ainda assim se tornou um gargalo para o aumento da eficiência do ParaSimTop. Considerando estas condições, após os resultados que se referem à simulação de 60 casos de contingencias diferentes, é possível se verificar que foram alcançados os objetivos, pois em todos eles:

1) As topologias que apresentavam pontos de sobrecarga foram descartadas da classificação;

b) As topologias que possuíam maior quantidade de pontos próximos aos limites referentes à sobrecarga e subtensão obtiveram classificações inferiores aos demais;

c) Os casos em que existiram empate nos critérios citados nos itens "a" e "b", o sistema possibilitou realizar a classificação topológica em função do número de manobras que cada topologia indicava para completar-se. Estes resultados possibilitaram o ParaSimTop entrar em uma nova fase que é o estudo de sua instalação para funcionamento online e este processo passará pelo aprimoramento de novos métodos de cálculos do fluxo de carga o que ofereceria um tempo de resposta menor aos operadores do sistema Elétrico e maior eficiência em uma analise comparativa. 


\section{CONCLUSÃO}

Neste trabalho apresentamos um Software Simulador de seleção de topologias denominado de ParaSimTop construído, em sua maior parte, com algoritmos fundamentados em Lógica Paraconsistente Anotada com anotação de dois valores (LPA2v). Apesar de estar em sua primeira versão o ParaSimTop é uma ferramenta computacional que possibilita um eficiente apoio às decisões de operadores em situações que exigem manobras de recomposição, bem como em simulações de contingencias de diversos tipos. O ParaSimTop também pode ser utilizado pela equipe de programação para estudos da melhor alternativa de configuração de rede em entrega programada de equipamentos, de forma a aperfeiçoar as condições de carregamento, níveis de tensão e confiabilidade do sistema de subtransmissão. Conforme visto nos resultados alcançados, está técnica fundamentada em Lógica Paraconsistente se mostrou bastante eficaz e futuramente outras pesquisas irão agregar novas característica ao ParaSimTop deixando-o mais pratico e confiável em tomadas de decisão da operação frente a eminentes mudanças de topologias em sistemas de subtransmissão de energia elétrica.

\section{REFERÊNCIAS}

[1] CIGRE, "Practical use of expert systems in planning and operation of power systems", TF 38.06.03, Électra, n.146, pp.30-67, Feb. 1993

[2] P. N.M.Dorantes, J. P. N. González, G.M.Mendez, " Fault Detection Systems via a Novel hybrid Methodology for Fuzzy Logic Systems based on Individual base inference and Statistical Process Control. IEEE Latin A merica Transactions, Vol. 12, No. 4, 2014.

[3] J. .I. Da Silva Filho, A. \& Rocco, "Power systems outage possibilities analysis by Paraconsistent Logic" Power and Energy Society General Meeting - Conversion and Delivery of Electrical Energy in the 21st Century, IEEE ISBN: 978-1-4244-1905-0, ISSN: $1932-5517 \mathrm{pp}(1-6)$ Pittsburgh, PA, 2008.

[4] M. T. P. de Leão, P. M. D. O. Jesús, "Comparative Analysis of Different Cost Loss Allocation Methodologies in Distribution Networks with Distributed Generation", Latin America Transactions, IEEE, vol.3, $\mathrm{n}^{\circ} .3$, pp. 290-295, 2005.

[5] M. R. A. Calado, S. J. P. S. Mariano, "Determination of the Earth Fault Factor in Power Systems for Different Earthed Neutrals", IEEE Latin America Transactions, Vol. 8, No. 6, pg. 637-645, December 2010.

[6] N.C.A. Da Costa \&D., Marconi, "An overview of Paraconsistent logic in the 80's., The Journal of Non-Classical L ogic, v.6, p. 5-31, 1989.

[7] V.S. Subrahmanian, "On the semantics of quantitative logic programs. Proc. 4th. IEEE Symposium on Logic Programming, Computer Society Press, Washington D.C, 1987.

[8] J. I. Da Silva Fiho, G. Lambert-Torres, G. and J. M. Abe. "Uncertainty Treatment Using Paraconsistent Logic - Introducing Paraconsistent Artificial Neural Networks.. IOS Press, p.328 pp.. Volume 211Frontiers in Artificial Intelligence and Applications, Amsterdam, Netherlands, 2010.

[9] L. F. P Ferrara, J. I. Da Silva Filho, A. Rocco, G. Lambert-Torres, M. R. Santos, F. B.Blanco," Forescaster of Loads Profile in Power Electric System based on Paraconsistent Annotated Logic. In: 15th Iternational Conference on Inteligent System Application to Power Systems - ISAP 2009, 2009, Curitiba. Proceedings of the 15th Iternational Conference on Inteligent System Application to Power Systems, 2009.

[10] J. I. Da Silva Filho, A. S. Onuki, L.F. P. Ferrara, M.C, .Mário, J. M. Camargo, D. V. Garcia,; M. R. Santos, A. Rocco,. Electric Power System Operation Decision Support by Expert System Built with Paraconsistent Annotated Logic. In: Petrica Vizureanu. (Org.). Advances in Expert Systems. 1ed.Rijeka: InTech, 2012, v. , p. 29-59

[11] L. F. P. Ferrara, J. M. Camargo, A. Rocco, J. I. Da Silva Filho, A. S Onuki., M. R. Santos, , G. Lambert-Torres, "Para-Previsor LPA2v: Um Sistema Computacional Previsor de Perfil de Cargas em Sistemas Elétricos de Potência construído com os fundamentos da Lógica Paraconsistente Anotada. Seleção Documental, v. 21, p. 11-17, 2011.

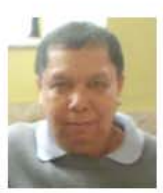

João Inácio Da Silva Filho é graduado em Engenharia Elétrica pela Universidade Santa Cecília (UNISANTA), Santos, SP, Brasil, em 1982. Obteve o título de mestre em Engenharia Elétrica pela Escola Politécnica da Universidade Estadual de São Paulo (POLI/USP) em 1999, e de Doutor, em Engenharia Elétrica pela mesma Instituição, em 2001. Atualmente é professor/pesquisador do Laboratório de Lógica Paraconsistente Aplicada - LaboLPA na Unisanta, em Santos-SP.

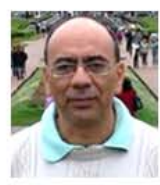

José de Melo Camargo é graduado em Engenharia Elétrica pela Universidade de Moji das Cruzes(1985), e fez especialização em Automação e controle em linhas, subestações e distribuição pelo Centro de Excelência - POLI - IEE- ELET.- CESP(1994). Atualmente é Engenheiro de Projetos Senior da AES Eletropaulo SA em São Paulo, SP,

Brasil.

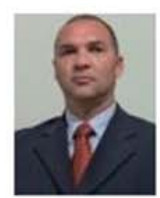

Marcos Rosa dos Santos Possui graduação em Engenharia Elétrica com ênfase Eletrônica pelo Centro Universitário da FEI (2003). Concluiu o Mestrado em Sistemas de Potência pela Escola Politécnica da USP (2008) e o Doutorado em Sistemas de Potência pela mesma Instituição, em 2013. Trabalha com Operação de Sistema de Potência em Tempo Real na AES Eletropaulo SA. Atualmente pesquisa novas técnicas de operação de tempo real com análises de paralelismos e efeitos transitórios.

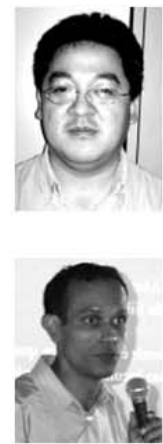

Alexandre Shozo Onuki é graduado em engenharia elétrica pela Universidade Santa Cecília, UNISANTA, Santos SP, Brasil e fez mestrado em Engenharia Elétrica pela Universidade Federal de Uberlândia UFU em Minas Gerais, Brasil, na área de Sistemas de Elétricos. Atualmente elabora pesquisas de aplicações na área de Sistemas Elétricos de Potência e técnicas de IA aplicadas em eng enharia elétrica.

Mauricio Conceição Mario é graduado em Engenharia Elétrica pela FEI - Faculdade de Engenharia Elétrica de São B. do Campo - SP, Brasil e Mestre em processamento da Informação pela Universidade Federal de Uberlândia - UFU em Minas Gerais. Em 2002 doutorou-se em Ciências pela Faculdade de Medicina da Universidade de São Paulo FMUSP em 2003. Atualmente é professor/pesquisador da Universidade Santa Cecília UNISANTA - em Santos SP.

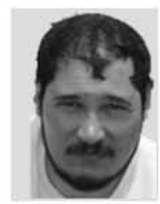

Luís Fernando Pompeo Ferrara é graduado em Engenharia Civil e Processamento de Dados pela Universidade Presbiteriana Mackenzie -SP Brasil. Obteve o título de Mestre em Engenharia Elétrica com ênfase em Processamento da Informação pela Universidade Federal de Uberlândia - UFU em Minas Gerais. Atualmente faz pesquisas de aplicações das Lógicas Não-Clássicas em automação e robótica.

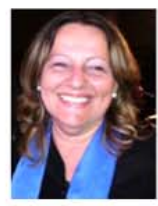

Dorotéa Vilanova Garcia é graduada em Matemática pela Faculdade de Filosofia Ciências e Letras de Santo André (1978), SP, Brasil. Concluiu Mestrado em Inteligência Artificial pela Universidade Federal de Uberlândia UFU MG, Brasil. Atualmente é docentes do Mestrado Profissional da Mecânica da UNISANTA- em Santos-SP, Brasil e faz pesquisas em aplicações de Lógicas não clássicas.

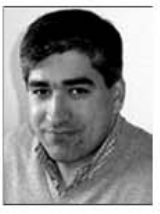

Jorge Manuel Correia Pereira é graduado em Matemática Aplicada para Ciência da Computação pela Faculdade de Ciências da Universidade do Porto -PT em 1991. Em 1995 obteve o grau de Mestre em Engenharia Elétrica pela mesma Universidade e em 1999 completou o seu doutoramento. Atualmente é professor pesquisador do INESC Porto, em Portugal.

Alexandre Rocco é graduado em Engenharia Elétrica pelo Centro

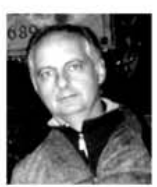

Universitário da FEI (1978). Fez mestrado em Automação de Sistemas Elétricos de Potência pela Universidade de Campinas -UNICAMP e doutoramento em Engenharia Elétrica na Escola Politécnica de São Paulo POLI/USP. Atualmente se dedica a pesquisas em áreas ligadas a geração e distribuição de Energ ia Elétrica. 